\title{
Neuroprotective Effects of 1,8-Cineole on Apoptosis Inhibition and Bcl-2/Caspase-3 mRNA Expression in the Hippocampus of Epileptic Pilocarpine Model Rats
}

\author{
Mahsa Nikbakht Rad ${ }^{1(D)}$, Iraj Jafari Anarkooli ${ }^{1 *(D)}$, Alireza Abdanipour ${ }^{1}(\mathbb{D}$
}

\section{Dept. of Anatomy, School of Medicine, Zanjan University of Medical Sciences, Zanjan, Iran}

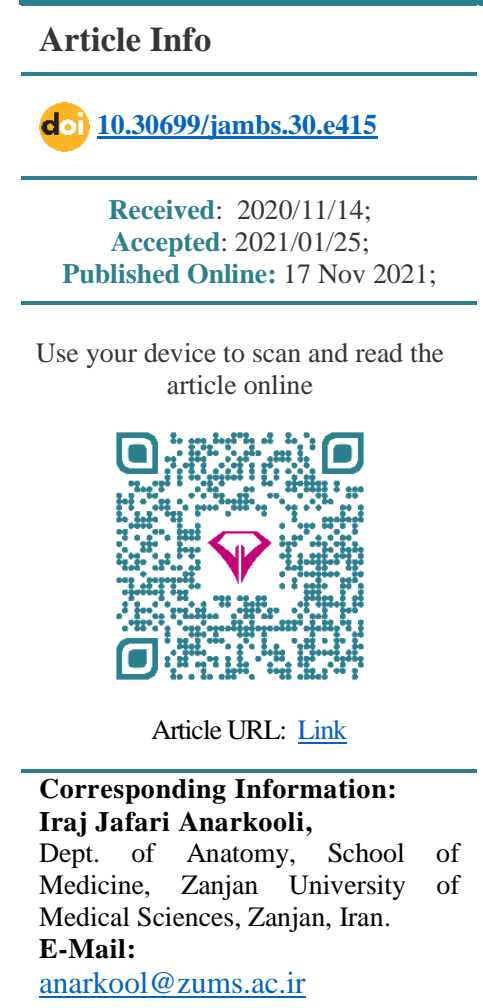

\begin{abstract}
Background \& Objective: The neuroprotective potential of 1,8-Cineole (CIN) has recently been documented in vitro. Here we studied potential beneficial therapeutic effects of CIN, using the temporal lobe epilepsy (TLE) pilocarpine rat model through up-regulation of $B c l-2$, as an anti-apoptotic gene.
\end{abstract}

Materials \& Methods: A total of 32 (n=8 per group) male Wistar rats were divided into 4 groups as follows: i) normal rats (received CIN (50 mg/kg)). ii) Non-treated epileptic rats. iii) Vehicle epileptic rats treated with 10\% Polysorbate 20 (Tween 20). iv) TLE-treated rats with CIN once daily $(50 \mathrm{mg} / \mathrm{kg})$, three days after the first seizure and up to 28 days, four days a week (treatment group).

For the analysis, based on the Racine scale, the score of 4 and 5 was chosen. Rats were sacrificed and primed 28 days after the first seizure for both histopathological and quantitative real time PCR (qRT-PCR) analysis.

Results: The findings showed that CIN prevents cell death caused by Pilocarpine, via regulatory effect on apoptotic and anti-apoptotic gene expression. QRT-PCR results showed a significant increment in the $\mathrm{Bcl}-2$ expression, and a decrease in Caspas-3 gene in the epileptic group treated with CIN. Also, amount of total antioxidant capacity was higher in CIN treated group. Histological study of the brain regions revealed a significant decrease in the apoptotic and necrotic hippocampal cells in the treatment groups.

Conclusion: Collectively, the present study showed CIN significantly induced neuroprotection effects for brain damage. It seems CIN can be a promising method for improving the effectiveness of therapy.

Keywords: 1,8-Cineole, Epilepsy, Pilocarpine, Bcl-2, Caspase-3

\section{(4) (6)

\section{Introduction}

Epilepsy is a chronic condition of the nervous system that affects people of all ages. As one of the most prevalent neurological disorders in the world, epilepsy affects around 50 million people worldwide (1). It is characterized by multiple epileptic seizures, which are caused by repeated abnormal and simultaneous electrical discharges, in a group of nerve cells in an area of the brain (2). Although there are several types of epilepsy, Temporal Lobe Epilepsy (TLE) is considered a more severe form in humans. Hippocampal sclerosis, degeneration of the nervous system and extensive reorganization of the hippocampal networks are among the neuropathological modifications reported in TLE individuals (3).

Generally, brain damage from epilepsy is a dynamic process. In epilepsy, multiple factors lead to brain neurons death (4); these include genetic, oxidative stress, mitochondrial dysfunction, and ROS factors (5). TLE is related to neuropathological changes, including hippocampal sclerosis and degeneration of neurons, and vast rearrangement of hippocampal circuits (6). Thus, it is necessary to create complementary therapeutic approaches, that can prevent the acute status epilepticus (SE) transition to the chronic (7).

Researchers consider the pilocarpine rat model to be the most widely recognized model for human epilepsy (8). The relationship between oxidative stress and the production of active oxygen species with epilepsy has been proven (4). Oxidative stress and mitochondrial dysfunction caused by reactive oxygen species (ROS) have been reported as effective factors in the pathophysiology of neurodegenerative disorders (9). Seizures destroy neuron mitochondria in the rat hippocampus, leading to loss of hippocampal neurons. 
The mitochondrial damage of hippocampal neurons in epileptic rats is closely related to the expression of $\mathrm{Fas}$ and Caspase-3(10).

Anti-epileptic drugs are the main form of treatment (9). Targeting Caspase-3 upper and lower cascade activities can also be used as a clinical target for epilepsy therapy (11). Usage of antioxidant agents or biomolecules that decrease apoptotic genes is a method to reduce neuronal cell death (apoptosis/necrosis). There are usually two main pathways for apoptosis: The $B c l-2$ family and the intrinsic or mitochondrial pathway mediated by the members of the Bak / Bax pathway. Bax protein increases mitochondrial membrane permeability and inhibits Bcl-2 (12). The external apoptosis pathway, which is initiated by the TNF family receptors, resulted in the triggering of Caspase- 8 and subsequently Caspase-3 (13). Based on the results of studies, oxidative stress plays a major role in epilepsy pathogenesis (14).

Recently, drug therapy has been the main treatment for epilepsy, and more than 10 forms of first-line antiepileptic drugs are available in hospitals. However, multiple forms of epilepsy cannot be adequately managed, creating a significant financial burden for both patients and society (15). CIN, a monoterpenic oxide is found in the leaves of many herbs, including eucalyptus; it has been used as an additive for food, as well as a drug for the improvement of symptoms in the infectious respiratory diseases (16).

Eucalyptus has also been used in the pharmaceutical industry to make various pharmaceutical formulations. In the previous studies, its inhibitory properties have been shown in the production of anti-inflammatory cytokines, such as prostaglandins and leukotrienes. It has also antimicrobial, anticancer, hepatoprotective, analgesic and anti-inflammatory properties. In addition to the above, it has antioxidant effects (17).

Interestingly, some antioxidant molecules were reported to decrease the levels of oxidative stress by scavenging ROS (18). Antioxidants alleviate the oxidative stress; they have increasingly become headlines in epilepsy treatment (19). In this study, we aimedto investigate the antioxidant effect and neuroprotection of CIN by examining the Bcl-2 and Caspase genes in pilocarpine epileptic rat model.

\section{Materials and Methods}

\section{Animal study design}

The Ethics Committee of Zanjan University of Medical Sciences has approved all procedures related to animal treatment and use (Ethics code: ZUMS.REC.1394.29). In the present study, 32 rats were divided into 4 main groups ( $n=8$ animals for each group). The details of each group are as follows. i) normal rats (received CIN (50 mg/kg)). ii) non-treated epileptic rats; iii) vehicle epileptic rats treated with $10 \%$ Polysorbate 20 (Tween 20). and iv) TLE-treated rats with CIN once daily $(50 \mathrm{mg} / \mathrm{kg}$ ), three days after the first seizure and up to 28 days, four days a week (treatment group).

\section{Pilocarpine Rat Model}

Thirty-two adult Wistar male rats were housed in room temperature $(\mathrm{RT})\left(21-22^{\circ} \mathrm{C}\right)$, under regulated routine conditions consisting of standard 12-hour light/dark cycle, and $45-55 \%$ humidity. Access to food and water was given to Rats. The mean weight was $250-300 \mathrm{~g}$.

Pilocarpine rat model of epilepsy was used for this study, as described previously. In short, the rats were given methylscopolamine bromide (1 $\mathrm{mg} / \mathrm{kg}$ s.c.) 30 minutes before the pilocarpine injection, to reduce the side effects of the IP injection of pilocarpine hydrochloride (350-400 mg/kg). An injection of 2,5 mg/kg of diazepam was administered to the affected rat an hour after the first epileptic occurrence. To assess the epileptic rats, we performed the Racine behavioral test.

Animals with the Racine score of 4 and 5 were chosen (20). They were sacrificed after 28 days for tissue experiments and gene expression study. Researchers also suggested that structural alterations in the hippocampus and changes in the gene expression arise within four weeks of epileptic seizure initiation (21).

\section{TUNEL and Acridine orange (AO) staining}

The animals were anesthetized with KetamineXylazine (44 and $13 \mathrm{mg} / \mathrm{kg}$ ) before scarifying, 28 days after the first SE (after the last injection of CIN). Trans cardiac perfusion was carried out with $20 \mathrm{ml}$ of heparinized saline and in PBS with $4 \%$ paraformaldehyde (pH 7.4). Over the next 24 hours, the brain tissue was impregnated with the same fixative. For each animal, we picked one hemisphere of 10 different sections, $7 \mu \mathrm{m}$ thick and located 2800-3800 $\mathrm{mm}$ from Bergman for histological assessment by AO and TUNEL staining. The different areas of which are shown in Figure 1.

Cell morphological necrosis changes were assessed by AO staining. In the ethanol solution with different dilutions $(50 \%, 70 \%, 85 \%, 95 \%$, and $100 \%)$ the paraffin components were dehydrated. The sections were then permeabilized by incubation in the PBS with \% 0.1 Triton $\mathrm{X}-100$ and $\% 0.1$ sodium citrate for 15 minutes, and 20 $\mu \mathrm{g} / \mathrm{mL}$ proteinase $\mathrm{K}$ for 15 minutes (22). For AO staining, the sections were incubated in $6 \mu \mathrm{g} / \mathrm{mL}$ AO (Sigma) in 0.1 $\mathrm{M}$ citric acid and, $0.2 \mathrm{M} \mathrm{Na} 2 \mathrm{HPO} 4(\mathrm{pH} 2.6$ ) for 30 minutes at RT.

Under a fluorescent microscope, the stained segments were observed. In this step, 200 cells were counted and the number of orange/yellow stained cytoplasm necrotic cells was measured. The nuclear necrotic cells had condensed chromatin and fragmented nuclei, which resembled viable cells. In order to stain the TUNEL, the sections were assisted using the In Situ Cell Death Detection kit (Roche, Germany), as directed by the manufacturer. TUNELpositive cells were exposed to 3, 3'-diaminobenzidine tetrahydrochloride (DAB; Sigma-Aldrich, Germany) chromogenic and hematoxylin-contained. Quantification of TUNEL-positive cells was assessed in all regions of the hippocampus (CA1, CA2, CA3, and CA4). Neuronal apoptosis was determined by the TUNEL method. 


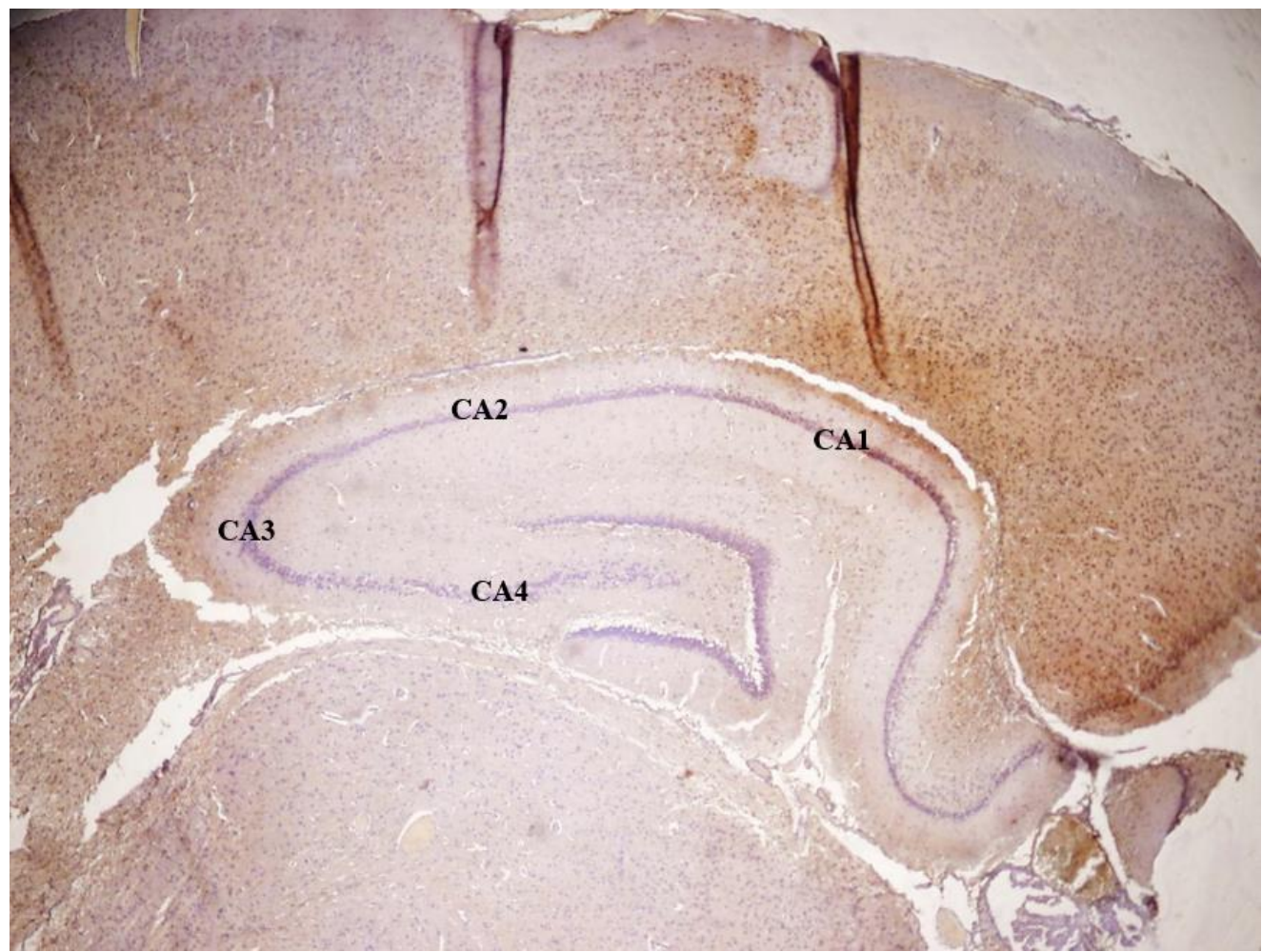

Figure 1: Different areas of the hippocampus

\section{Real-time PCR}

Real-time PCR (RT-PCR) was done for all the experimental groups using prepared cDNA. TRIzol ${ }^{\circledR}$ (Invitrogen / Life Technologies) was used to isolate total RNA from hippocampal tissues. Following the manufacturer's guidance, $1000 \mathrm{ng}$ of purified RNA was used to synthesize $20 \mu \mathrm{L}$ of cDNA, according to the RevertAid $^{\mathrm{TM}}$ First Strand cDNA Synthesis Kit (Fermentas, Germany). The mRNA levels of Bcl-2 and Caspase-3 neurotrophins were quantified using cDNA.
As an internal normalization control, glyceraldehyde 3phosphate dehydrogenase (GAPDH) was used (Table 1). PCR reactions were performed in a $12.5 \mu \mathrm{L}$ final reaction volume containing forward and reverse primers $(200 \mathrm{nM}$ each), cDNA $(0.5 \mu \mathrm{L} ; 25 \mathrm{ng}$ of RNA samples), SYBR® Green I $(6.5 \mu \mathrm{L}$; Fermentas; Thermo Fisher Scientific, Inc.) and nuclease-free water, up to final volume for 40 cycles; they were done at $95^{\circ} \mathrm{C}$ for 15 s followed by $60^{\circ} \mathrm{C}$ for 1 minute. Using the $\Delta \Delta \mathrm{Cq}$ method, relative changes in target mRNA levels were calculated (23).

Table 1: Primer parameters and Gen Bank accession number.

\begin{tabular}{cccccc} 
Gene & Accession no. & Sense 5' $\rightarrow \mathbf{3}^{\prime}$ & Anti-sense $\mathbf{5}^{\prime} \rightarrow \mathbf{3}^{\prime}$ & $\mathbf{b p}$ & Tm \\
\hline Caspase-3 & NM_012922.2 & CGCACCCGGTTACTATTCCTGG & TGGCCACCTTCCGGTTAACAC & 137 & 60 \\
Bcl-2 & NM_016993.1 & GTGGCCTTCTTTGAGTTCGGTG & ATCCCAGCCTCCGTTATCCTG & 147 & 60 \\
\hline GAPDH & NM_017008.4 & TTGTCAGCAATGCATCCTGCAC & GTCTGGGATGGAATTGTGAG & 158 & 60 \\
\hline
\end{tabular}

Primers were designed, using the GENE RUNNER Version 3.05; it is produced by Genfanavaran Company. Caspase3, cysteine-aspartic acid protease3; Bcl-2: B-cell lymphoma 2; GAPDH: glyceraldehyde-3-phosphate dehydrogenase.

\section{Evaluation of Total Antioxidant Capacity (TAC) of the Hippocampus}

Hippocampi TAC assessment was performed using plasma reduction capacity ferric (FRAP) assay. This assay is based on the reduction of tripyridyl-s-triazine (TPTZ). TPTZ complex is a coloring ligand, which produces a deep blue color. To prepare the FRAP solution, 3 compounds of acetate $(300 \mathrm{mM})$ iron (III) chloride (20 $\mathrm{mM})$, and TPTZ (10 $\mathrm{mM})$ were combined.
Various concentrations $(0,125,250,500,750$ and 1000 $\mu \mathrm{M}$ ) of iron (II) phosphate was diagramed for the typical FRAP curve. Sample supernatant were mixed with a 1.5 $\mathrm{ml}$ FRAP reagent. The wavelength was read at $593 \mathrm{~nm}$. By the use of standard curve, data were reported as $\mu \mathrm{M}$ of iron (II) per $g$ of the sample (24).

\section{Statistics}

Statistical analysis was conducted by the SPSS software v.15. Independent experiments were replicated 
five times; the data have been shown as a standard mean error (SEM). One-way ANOVA was used to analysis the data between the groups, followed by post hoc from Tukey $(\mathrm{P}<0.05)$.

\section{Results}

\section{Pilocarpine-treated rats}

The rats exhibited peripheral side effects of cholinergic symptoms, including diarrhea, salivation, and lacrimal gland activation, 5 minutes after the pilocarpine injection. The rats displayed the signs of head nodding, scraping, masticatory jaw motions, chewing, and exploratory actions over the following 15-20 minutes. Recurrent seizures began approximately $38.42 \pm 7.29$ minutes after the pilocarpine injection. Approximately $72 \%$ of the animals exhibited seizures after the pilocarpine injection. A seizure-free phase (latent) was followed by the initial acute insult and eventually a chronic period.

\section{Detection of apoptotic cells (TUNEL assay)}

Figures $2 \mathrm{~A}$ and 3 show the results. In the CA1, CA2, $\mathrm{CA} 3$ and CA4 regions of the hippocampus, the percentage of necrotic cells was calculated. Figure 2 indicates the average number of necrotic cells in CA1 treated with CIN $(30.76 \pm 2.31), \mathrm{CA} 2(32.93 \pm 3.14), \mathrm{CA} 3$ (33.85 \pm 1.79$)$, and CA4 $(24.71 \pm 2.38)$ groups, as well as for the nontreated CA1 $(44.41 \pm 1.78)$, CA2 $(46.54 \pm 2.27)$, CA3 $(50.85 \pm 1.78)$, and CA4 (45.11 \pm 1.31$)$ groups. Compared to non-treated groups, apoptotic cells significantly decreased in all regions in the CIN treated groups $(\mathrm{P}<0.05)$. Figure 3 shows TUNEL staining of the cells and hippocampal tissues.

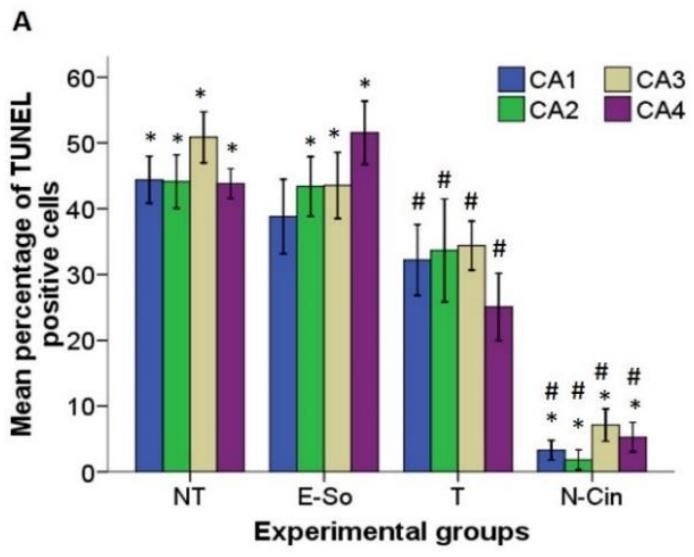

B

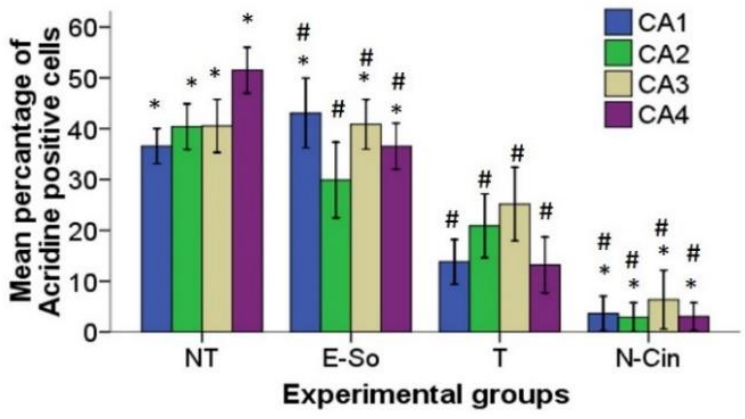

Figure 2: The results TUNEL and AO assays. The mean percentage of apoptotic (A) and necrotic (B) cells. In 4 regions (CA1, CA2, CA3 and CA4) of the hippocampus, apoptotic and necrotic cells were recorded. The mean \pm SEM is shown by the bars; *compared to CIN treated group; \#compared to non-treated group; $\mathbf{P}<0.05$. NT: non-treated epileptic rats; E-So: epileptic rats treated with 10\% Polysorbate 20; T: Epileptic rats treated with CIN once daily; N-Cin: normal rats (received CIN).

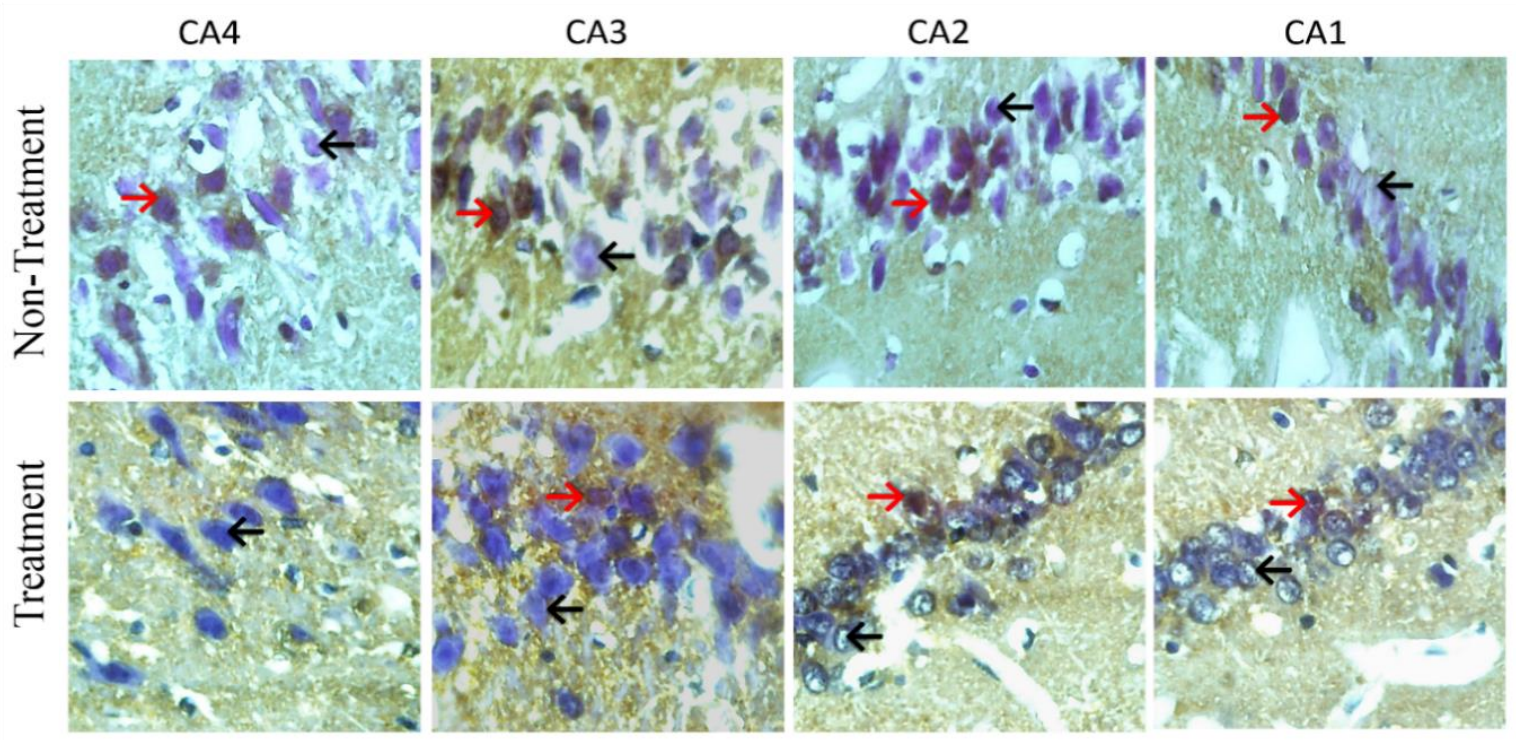

Figure 3: Representative images of TUNEL and Hematoxylin stains in the hippocampus regions (CA1, CA2, CA3 and CA4), In experimental groups, photomicrographs displayed positive TUNEL cells. DNA fragmentation and non-fragmentation are indicated by red and black arrows. Hematoxylin was utilized for counterstaining. Magnification: 200-fold. Non-treated: nontreated epileptic rats; Treatment: Epileptic rats treated with CIN once daily. 


\section{Necrotic cell identification (Acridine staining)}

The percentage of necrotic cells was calculated in the CA1, CA2, CA3, and CA4 regions of the hippocampus. Figure 2 shows the mean percentage of necrotic cells in the CIN treated CA1 $(17.32 \pm 2.31)$, CA2 $(21.71 \pm 2.10)$, CA3 $(26.45 \pm 3.57)$, and CA4 $(16.73 \pm 2.59)$ groups, and non-treated (CA1 $(36.57 \pm 1.72), \mathrm{CA} 2(40.40 \pm 2.23)$, CA3 $(41.14 \pm 2.26)$, and CA4 $(48.36 \pm 1.52)$ groups. The necrotic cell significantly decreased in the CA3 and CA4 regions in the $\mathrm{CIN}$ - treated groups, compared to nontreated groups $(\mathrm{P}<0.05)$ (Figures $2 \mathrm{~B}$ and 4$)$.

\section{Gene expression}

Changes in Bcl-2 and Caspase-3 mRNA expressions in test groups were studied using quantitative real-time PCR
(qRT-PCR). The findings have been presented independently compared to the normal group. The CIN treatment group (relative to the normal group) had significantly increased expression of $\mathrm{Bcl}-2(3.351 \pm 0.35)$ compared to non-treated $(0.13 \pm 0.00)$ and vehicles groups. Also, the treatment group had decreased expression of Caspase-3 $(0.13 \pm 0.05)$ compared to other experimental groups (Figure 5).

\section{TAC Evaluation}

The results of the TAC assay showed a significant difference in the antioxidant activity level between the different groups. The amount of TAC was higher in CIN treated groups compared to non-treated groups; the difference was significant $(\mathrm{P}<0.001)$ (Figure 6).
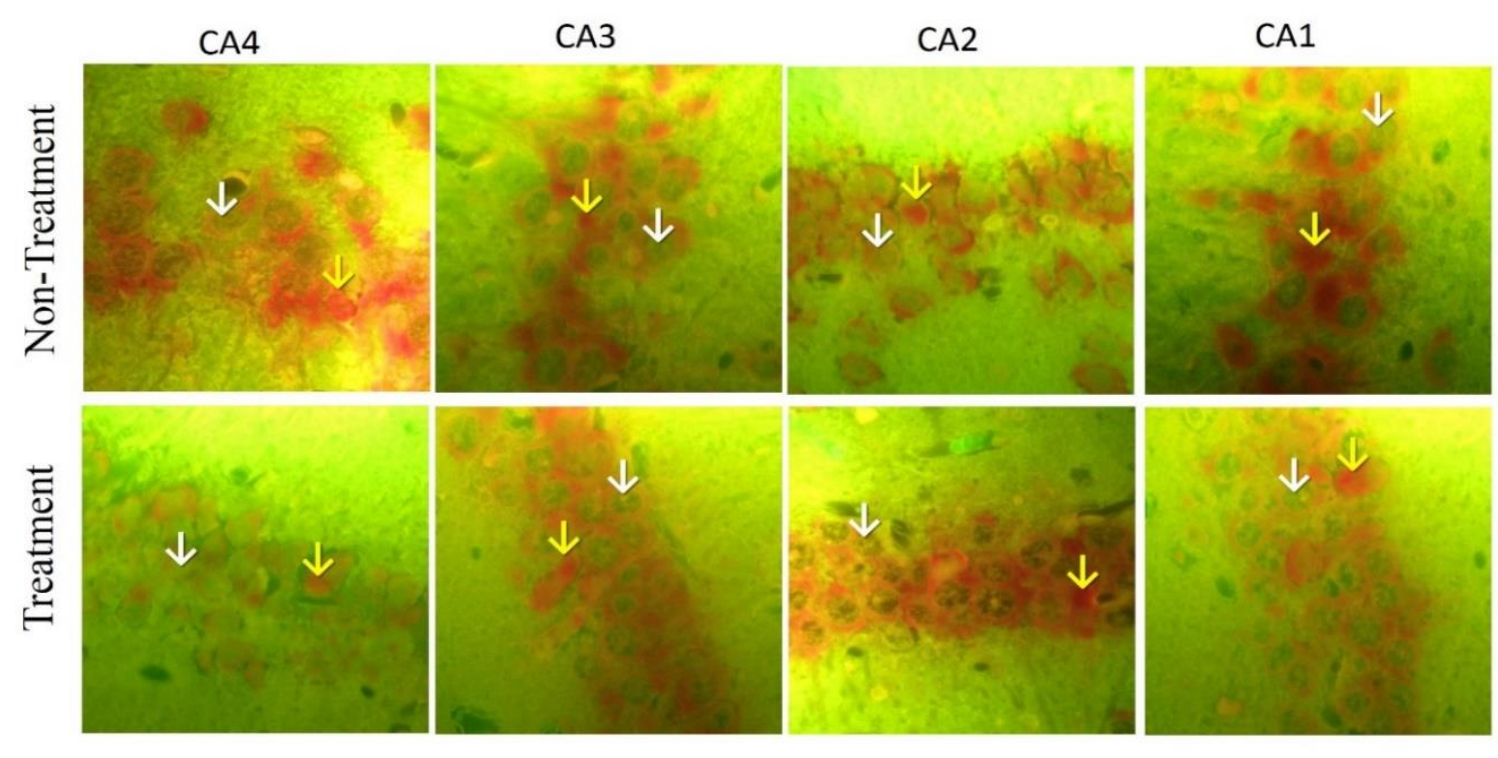

Figure 4: Acridine staining representative images in the regions of hippocampus. Photomicrographs of positive Acridine cells in the experimental groups. Yellow and white arrows respectively show death and intact cells. Magnification: 200-fold. Non-Treated: non-treated epileptic rats; Treatment: Epileptic rats treated with CIN once daily.
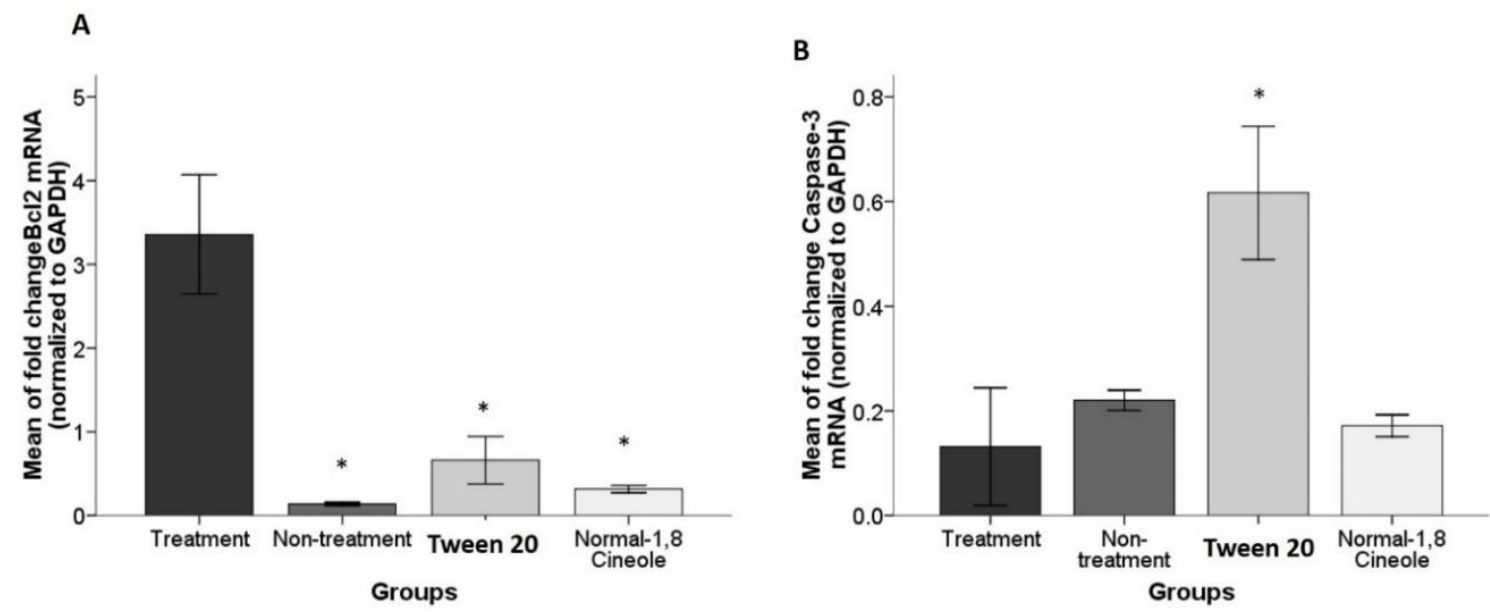

Figure 5: qRT-PCR performance, relative to the normal group. The mRNA level of Bcl-2 (A) and Caspase-3 (B) Quantitative gene expression data were normalized to the expression levels of GAPDH gene. The mean \pm SEM is shown by the bars; * (compared to treated group), $\mathrm{P}<0.05$. 


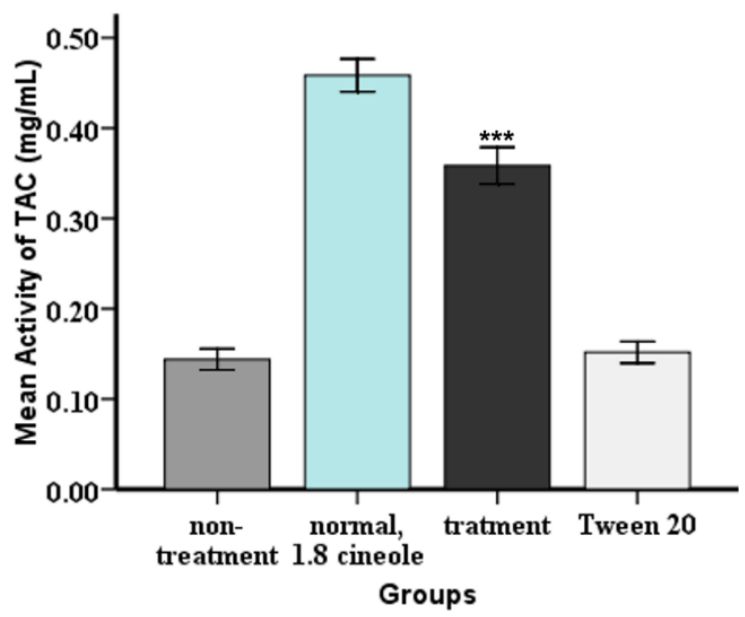

Figure 6: The effects of CIN on TAC activity in rats' hippocampi. The mean \pm SEM is shown by the bars. $* * * P<0001$ (compared to other groups).

\section{Discussion}

In the present study, our findings showed a considerable increase in $\mathrm{Bcl}$-2 mRNA expression of the CIN treated epileptic rats. However, the mRNA expression of Caspase-3 decreased relative to the intact rats. Attained results from the present study indicate that CIN treatment could reduce the number of apoptotic neurons in hippocampal regions of the pilocarpinetreated rats. Also, the results of TAC assay of the studied groups showed the improvement of antioxidant activity in the CIN-treated groups.

Our finding of the tissue assessment in this study showed, that epileptic seizure causes apoptosis and necrosis of a large number of neural cells in different regions of the hippocampus. One of the main causes of the pathogenesis of TLE is apoptosis of neural cells in the brain regions, especially the hippocampal area (25).

Oxidative stress is recommended as a factor to monitor aging and various neuropathies. Excess oxidants cause antioxidant deficiencies, resulting in redox imbalances in the body. Oxidative exposure is one of the causes of neurodegenerative diseases (26). The brain is vulnerable to oxidative stress due to the high content of polyunsaturated fatty acids and high oxygen demand. The imbalance between ROS production and degradation initiates oxidative stress (27).

Oxidative stress and mitochondrial dysfunction have a crucial influence on seizure start in epileptic patients (25). The mitochondria role in TLE is very important. Free radicals can be produced during brain damage induced by seizures; overproduction of free radicals can damage cellular components (28). Free radicals, in the presence of $\mathrm{Fe} 2+$ and $\mathrm{Cu} 2+$, can oxidize DNA and essential biomolecules, and increase the excitability of the neuron for epileptic seizure. Antioxidants can neutralize the free radicals, in order to remove the unpaired state of the radicals by accepting or donating electron(s) (29).

The TAC in epileptic group treated with CIN was more than non-treated group; it indicates the supportive effect of CIN on the neurons in the hippocampal region.
Researches have shown, that CIN can effectively cross the blood-brain barrier by operating on receptor sites or enzyme structures; it can also have immediate brain effects (30). Previous studies have shown the antioxidant and neuroprotective effects of this compound (21). It is thought, that in our study CIN has an aiding effect on antioxidative system of the brain, which is seen in CIN treated group, in TAC assay test.

Considering the role of apoptosis in neurodegenerative diseases, preventing cell death might be one of the potential therapeutic targets by reducing the involved genes, such as Bcl-2 and Caspase-3; they can be regulated by drug therapy. Overproduction of ROS is followed by the Caspase-3 activation. It is reported, that CIN could inhibit intra-cellular ROS production and expression of catalytic antioxidant enzymes such as superoxide dismutase, glutathione peroxidase, and glutathione reductase. CIN may reduce apoptosis through reduced Caspase- 3 activity. It seems that the mechanism of its antioxidant action is through ROS scavenging and induction of nuclear $\mathrm{Nrf-2}$ factor (2).

Our main goal was to determine the beneficial effects of CIN in pilocarpine-induced TLE rats, and assess the alterations in apoptotic and anti-apoptotic mRNA expressions in the CA1-3 regions of the hippocampus. Studies on the analysis of brain samples of patients with TLE show, that the expression of $\mathrm{Bcl}-2$ and Caspase-3 differs in the hippocampus of these patients (31). A study was done on the kainic acid type of TLE rat model also showed that SE activates signaling factors, reducing the expression of $\mathrm{Bcl}-2$ four hours after the onset of SE; on the other hand, the expression of Caspase-3 is induced during 24-16 hours after the occurrence of SE increase (32).

Our analysis showed that neuronal apoptosis in the CA1-3 hippocampus region was evident, using TUNEL staining in TLE rat; it is in line with prior researches (19). TUNEL stain directly finds apoptotic cells, and the family of $\mathrm{Bcl}-2$ proteins regulates the apoptosis by 
adjusting the release of mitochondrial apoptosis factors, cytochrome c, and apoptosis (33). Previous experiments have shown, that Bax and Caspase-3 dramatically are stimulated in the brain of epilepsy rats, cleaved Caspase3 and pro-apoptotic Bax protein increases significantly, and anti-apoptotic $\mathrm{Bcl}-2$ protein decreases (34).

Our results confirmed these changes, and CIN reversed the changes. The regulatory protein category, such as anti-apoptotic $\mathrm{Bcl}-2, \mathrm{Bcl}-\mathrm{XL}$ and $\mathrm{Nrf} 2$ protects cells against apoptosis. Many apoptotic pathways are involved in the activation of Bcl-2, Bcl-XL and Nrf2 genes $(34,35)$. Analysis of the results of gene expression in this study showed an amplify in the expression of $\mathrm{Bcl}$ 2 in CIN treated groups, indicating the protective effect of this compound.

We also evaluated the effect of CIN on pilocarpineinduced neural loss in CA1-3 areas, via the TUNEL and AO assays. Our results demonstrated significantly decreased pilocarpine-induced cellular death in hippocampal regions in CIN-treated group. Our data provided preliminary evidence, suggesting CIN can protect a neuronal population from seizure-induced apoptotic cell death. The up-regulation of several antiapoptotic genes such as $\mathrm{Bcl}-2$ could result in certain inhibitory effects.

\section{Conclusion}

Thus, the present study has shown, that CIN has an important neuroprotective effect after pilocarpine-induced TLE neurological damage. It provides neuroprotection after delayed intervention after trauma; CIN prevents the onset of oxidative stress and the death of hippocampal neurons during TLE. It may be considered as an acceptable and lowcost candidate for treatment of epilepsy and other diseases of the nervous system. Further investigation is needed to confirm the findings of this report and to explore the underlying cellular and molecular mechanisms of the neurotrophin regulation by $\mathrm{CIN}$.

\section{Acknowledgment}

Present study was undertaken by Zanjan Medical College, Iran (Grant Number-A-12-20-8).

\section{Conflicts of Interest}

There is no conflict of interest.

\section{References}

1. Mameniskiene R, Karmonaite I, Zagorskis R. The burden of headache in people with epilepsy. Seizure. 2016 Oct 1;41:120-6. [DOI:10.1016/j.seizure.2016.07.018] [PMID]

2. Stafstrom CE, Carmant L. Seizures and epilepsy: an overview for neuroscientists. Cold Spring Harb Perspect Med. 2015 Jun 1;5(6).
[DOI:10.1101/cshperspect.a022426] [PMCID]

[PMID]

3. Losing P, Niturad CE, Harrer M, Reckendorf CMZ, Schatz T, Sinske D, et al. SRF modulates seizure occurrence, activity induced gene transcription and hippocampal circuit reorganization in the mouse pilocarpine epilepsy model. Mol Brain. 2017;10(1):30. [DOI:10.1186/s13041-017-0310-2] [PMID] [PMCID]

4. Aguiar CCT, Almeida AB, Araújo PVP, Abreu RNDCd, Chaves EMC, Vale OCd, et al. Oxidative stress and epilepsy: literature review. Oxid Med Cell Longev. 2012 Jul 14;2012. [DOI:10.1155/2012/795259] [PMID] [PMCID]

5. Shin EJ, Jeong JH, Chung YH, Kim WK, Ko KH, Bach JH, et al. Role of oxidative stress in epileptic seizures. Neurochem Int. 2011;59(2):122-37. [DOI:10.1016/j.neuint.2011.03.025] [PMID] [PMCID]

6. Thom M. Review: Hippocampal sclerosis in epilepsy: a neuropathology review. Neuropathol Appl Neurobiol. 2014;40(5):520-43. [DOI:10.1111/nan.12150] [PMID] [PMCID]

7. Xie C, Sun J, Qiao W, Lu D, Wei L, Na M, et al. Administration of Simvastatin after Kainic AcidInduced Status Epilepticus Restrains Chronic Temporal Lobe Epilepsy. PloS one. 2011;6(9):e24966.

[DOI:10.1371/journal.pone.0024966]

[PMID] [PMCID]

8. Vizuete AFK, Hansen F, Negri E, Leite MC, de Oliveira DL, Goncalves CA. Effects of dexamethasone on the Li-pilocarpine model of epilepsy: protection against hippocampal inflammation and astrogliosis. J Neuroinflammation. 2018;15(1):68. [DOI:10.1186/s 12974-018-1109-5] [PMID] [PMCID]

9. Hanaya R, Arita K. The New Antiepileptic Drugs: Their Neuropharmacology and Clinical Indications. Neurol Med Chir (Tokyo). 2016;56(5):205-20. [DOI:10.2176/nmc.ra.20150344] [PMID] [PMCID]

10. Taskiran AS, Ergul M, Gunes H, Ozturk A, Sahin B, Ozdemir EJC, et al. The effects of proton pump inhibitors (pantoprazole) on pentylenetetrazoleinduced epileptic seizures in rats and neurotoxicity in the SH-SY5Y human neuroblastoma cell line. Cell Mol Neurobiol. 2021 Jan;41(1):173-83. [DOI:10.1007/s10571-020-00956-6] [PMID]

11. Hussein AM, Eldosoky M, El-Shafey M, ElMesery M, Ali AN, Abbas KM, et al. Effects of metformin on apoptosis and $\alpha$-synuclein in a rat model of pentylenetetrazole-induced epilepsy. 
2019;97(1):37-46. [DOI:10.1139/cjpp-2018-0266] [PMID]

12. Kale J, Osterlund EJ, Andrews DW. BCL-2 family proteins: changing partners in the dance towards death. Cell Death Differ. 2018;25(1):65-80. [DOI:10.1038/cdd.2017.186] [PMID] [PMCID]

13. Elmore S. Apoptosis: a review of programmed cell death. Toxicol Pathol. 2007;35(4):495-516. [DOI:10.1080/01926230701320337] [PMID] [PMCID]

14. Pearson-Smith JN, Patel. Metabolic dysfunction and oxidative stress in epilepsy .Int J Mol Sci. 2017;18(11):2365. [DOI:10.3390/ijms18112365] [PMID] [PMCID]

15. Feng J, Feng L, Zhang GJE, medicine t. Mitochondrial damage in hippocampal neurons of rats with epileptic protein expression of Fas and caspase-3. Exp Ther Med. 2018;16(3):2483-9. [DOI:10.3892/etm.2018.6439] [PMID] [PMCID]

16. Santos FA, Silva RM, Campos AR, De Araujo RP, Lima Junior RC, Rao VS. 1,8-cineole (eucalyptol), a monoterpene oxide attenuates the colonic damage in rats on acute TNBS-colitis. Food Chem Toxicol. 2004;42(4):579-84. [DOI:10.1016/i.fct.2003.11.001] [PMID]

17. Ciftci O, Ozdemir I, Tanyildizi S, Yildiz S, Oguzturk HJT, health i. Antioxidative effects of curcumin, $\beta$-myrcene and 1, 8-cineole against 2, 3, 7, 8-tetrachlorodibenzo-p-dioxin-induced oxidative stress in rats liver. Toxicol Ind Health. 2011;27(5):447-53. [DOI:10.1177/0748233710388452] [PMID]

18. Kegler A, Caprara ALF, Pascotini ET, Arend J, Gabbi P, Duarte MM, et al. Apoptotic Markers Are Increased in Epilepsy Patients: A Relation with Manganese Superoxide Dismutase Ala16Val Polymorphism and Seizure Type through IL-1 $\beta$ and IL-6 Pathways. Biomed Res Int. 2020 Mar 6;2020. [DOI:10.1155/2020/6250429] [PMID] [PMCID]

19. Guo X, Wang J, Wang N, Mishra A, Li H, Liu H, et al. Wogonin preventive impact on hippocampal neurodegeneration, inflammation and cognitive defects in temporal lobe epilepsy. Saudi J Biol Sci. 2020 Aug 1;27(8):2149-56. [DOI:10.1016/j.sjbs.2020.05.030] [PMID] [PMCID]

20. Racine RJ. Modification of seizure activity by electrical stimulation. II. Motor seizure. Electroencephalogr Clin Neurophysiol. 1972 Mar 1;32(3):281-94.

[DOI: $10.1016 / 0013-$ 4694(72)90177-0]

21. Jongbloets BC, van Gassen KL, Kan AA, Olde Engberink AH, de Wit M, Wolterink-Donselaar IG, et al. Expression profiling after prolonged experimental febrile seizures in mice suggests structural remodeling in the hippocampus. PLoS One. 2015 Dec 18;10(12):e0145247 [DOI:10.1371/journal.pone.0145247] [PMID] [PMCID]

22. Yeh LK, Liu CY, Chien CL, Converse RL, Kao WW, Chen MS, et al. Molecular analysis and characterization of zebrafish keratocan (zKera) gene. J Biol Chem. 2008 Jan 4;283(1):506-17. [DOI:10.1074/jbc.M707656200] [PMID]

23. Xu L, Xu H, Cao Y, Yang P, Feng Y, Tang Y, et al. Validation of Reference Genes for Quantitative Real-Time PCR during Bicolor Tepal Development in Asiatic Hybrid Lilies (Lilium spp.). Front Plant Sci. 2017 Apr 25;8:669. [DOI:10.3389/fpls.2017.00669] [PMID] [PMCID]

24. Sayyar Z, Yazdinezhad A, Hassan M, Jafari Anarkooli I. Protective Effect of Matricaria chamomilla Ethanolic Extract on Hippocampal Neuron Damage in Rats Exposed to Formaldehyde. Oxid Med Cell Longev. 2018 Aug 14;2018. [DOI:10.1155/2018/6414317] [PMID] [PMCID]

25. Mendez-Armenta M, Nava-Ruiz C, JuarezRebollar D, Rodriguez-Martinez E, Gomez PY. Oxidative stress associated with neuronal apoptosis in experimental models of epilepsy. Oxid Med Cell Longev. 2014;2014:293689. [DOI:10.1155/2014/293689] [PMID] [PMCID]

26. Kim GH, Kim JE, Rhie SJ, Yoon S. The Role of Oxidative Stress in Neurodegenerative Diseases. Exp Neurobiol. 2015;24(4):325-40. [DOI:10.5607/en.2015.24.4.325] [PMID] [PMCID]

27. Chen C, Mei Q, Wang L, Feng X, Tao X, Qiu C, et al. TIGAR suppresses seizures induced by kainic acid through inhibiting oxidative stress and neuronal apoptosis. Biochem Biophys Res Commun. 2019 Jul 30;515(3):436-41. [DOI:10.1016/j.bbrc.2019.05.156] [PMID]

28. Puttachary S, Sharma S, Stark S, Thippeswamy T. Seizure-induced oxidative stress in temporal lobe epilepsy. BioMed research international. 2015 Jan 20;2015. [DOI:10.1155/2015/745613] [PMID] [PMCID]

29. Imam MU, Zhang S, Ma J, Wang H, Wang F. Antioxidants Mediate Both Iron Homeostasis and Oxidative Stress. Nutrients. 2017;9(7). [DOI:10.3390/nu9070671] [PMID] [PMCID]

30. Moss M, Oliver L. Plasma 1,8-cineole correlates with cognitive performance following exposure to rosemary essential oil aroma. Ther $\mathrm{Adv}$ Psychopharmacol. 2012;2(3):103-13. [DOI:10.1177/2045125312436573] [PMID] [PMCID]

31. Schindler CK, Shinoda S, Simon RP, Henshall DC. Subcellular distribution of Bcl-2 family proteins 
and 14-3-3 within the hippocampus during seizureinduced neuronal death in the rat. Neurosci Lett. 2004 Feb 19;356(3):163-6. [DOI:10.1016/j.neulet.2003.11.048] [PMID]

32. Mikati MA, Zeinieh M, Habib RA, El Hokayem J, Rahmeh A, El Sabban M, et al. Changes in sphingomyelinases, ceramide, $\operatorname{Bax}, \operatorname{Bcl}(2)$, and caspase-3 during and after experimental status epilepticus. Epilepsy Res. 2008;81(2-3):161-6. [DOI:10.1016/i.eplepsyres.2008.05.009] [PMID]

33. Lee J-M, Ji E-S, Kim T-W, Kim C-J, Shin M-S, Lim B-V, et al. Treadmill exercise improves memory function by inhibiting hippocampal apoptosis in pilocarpine-induced epileptic rats. J
Exerc Rehabil. $2018 \quad$ Oct;14(5):713. [DOI:10.12965/jer.36394.197] [PMID] [PMCID]

34. Li Q, Han Y, Du J, Jin H, Zhang J, Niu M, et al. Alterations of apoptosis and autophagy in developing brain of rats with epilepsy: Changes in LC3, P62, Beclin-1 and Bcl-2 levels. Neurosci Res. 2018 May 1;130:47-55. [DOI:10.1016/j.neures.2017.08.004] [PMID]

35. Anilkumar U, Prehn JHJFicn. Anti-apoptotic BCL-2 family proteins in acute neural injury. Front Cell Neurosci. $2014 \quad$ Sep 30;8:281. [DOI:10.3389/fncel.2014.00281] [PMID] [PMCID]

\section{How to Cite This Article:}

Nikbakht Rad M, Jafari Anarkooli I, Abdanipour A. Neuroprotective Effects of 1,8-Cineole on Apoptosis Inhibition and Bcl-2/Caspase-3 mRNA Expression in the Hippocampus of Epileptic Pilocarpine Model Rats. J Adv Med Biomed Res. 2022; 30 (138): 30-38

\section{Download citation:}

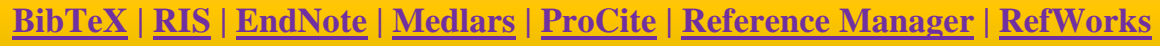

